\title{
Rare Cause of Post-Partum Spontaneous Paraplegia: Spontaneous Spinal Epidural Hematoma - MRI Findings a Case Report
}

\section{Authors \\ Dr Bhupendra Chaudhary ${ }^{1}$, Dr Seema Alwadhi ${ }^{2}$, Dr Supreethi Kohli ${ }^{3}$, Dr Sahaj Chopra², Dr Vikas Yadav²}

${ }^{1}$ Senior Resident, Department of Radio-Diagnosis, ESI Model Hospital \& PGIMSR, Basaidarapur, Delhi

${ }^{2}$ Specialist, Department of Radio-Diagnosis, ESI Model Hospital \& PGIMSR, Basaidarapur, Delhi

${ }^{3}$ Head of Dept, Department of Radio-Diagnosis, ESI Model Hospital \& PGIMSR, Basaidarapur, Delhi

\section{ABSTRACT}

Spontaneous spinal epidural hematoma (SSEH) is anextremely rare entity with incidence rate of 0.1 per 100,000 per year and causes sudden onset of neurological deficit in patient in post-partum period with no preceding underlying cause or intra partum cause resulting in spontaneous spinal epidural hematoma. We present a case of sudden onset paraplegia in post-partum period with on investigation shows epidural hematoma in dorsolumbar region with adjacent myelopathic changes. MRI is the investigation of choice to diagnose and to see the extent of lesion and help in planning further management of patient. Our patient went surgical removal of hematoma and in post operated period showed rapid reversal of neurological symptoms.

Keywords: Spontaneous spinal epidural hematoma, SSEH, post-partum paraplegia, sudden onset paraplegia

\section{INTRODUCTION}

Spontaneous spinal epidural hematoma (SSEH) is a rare cause of rapidly progressive neurological deficit so early diagnosis and management is necessary to prevent permanent disability. It is rare with incidence rate of 0.1 per 100,000 per year ${ }^{[1]}$. Common causes includes ${ }^{[2]}$ minor trauma, coagulopathy, anticoagulant therapy, vascular malformation, transient vascular hypertension, disc herniation and idiopathic causes. We present a case of acute onset paraplegia in post-partum status with no predisposing factor which was diagnosed on MRI as a case of epidural hematoma involving D12 to L2 level.

\section{CASE REPORT}

A 29 year old female patient was admitted in our hospital with acute onset paraplegia. She had given birth to her third child by normal vaginal delivery. Her antenatal course had been uneventful. There was no history of trauma, drug use or any physical exertion. On $3^{\text {rd }}$ postpartum day she experienced sudden onset lower backache with numbness in both lower limbs while urinating.Next morning she developed persistent backache and was unable to walk with subsequent involvement of bowel and bladder. Patient was admitted to casualty of our hospital.On admission she was conscious, well oriented, and afebrile with decreased tone of bilateral lower limbs and absent deep tendon reflexes at knee and ankle joint with sensory deficit below the level of L1. 


\section{JMSCR Vol||3||Issue||12||Page 8680-8683||December}

On investigation she had $\mathrm{Hb} \sim 10.9$, TLC of 9200, platelet count of $2.5 \mathrm{lk}, \mathrm{Na}^{+}, 128 \mathrm{mEq} \mathrm{Liter}{ }^{-1}$; $\mathrm{K}^{+}$, $4.3 \mathrm{mEq} \mathrm{Liter}{ }^{-1}$. On gynaecological examination per abdomen uterus is well involute with no bleeding or discharge with healthy lochia on postpartum $6^{\text {th }}$ day. On post-partum $8^{\text {th }}$ day Lumbar puncture was done at L3/4 level revealed normal opening pressure and drained xanthochromic fluid. Sugar was $45 \mathrm{mg} \mathrm{dl}^{-1}$, protein $185 \mathrm{mg} \mathrm{dl}^{-1}$, red blood cells $115 \mathrm{ml}^{-1}$, and white blood cells 2 . Patient was planned for MRI dorsolumbar spine and it reveals extra medullary biconvex lesion measuring 58x10 mm along $\mathrm{T} 11$ to $\mathrm{L} 1$ vertebra displacing the dura anteriorly and showing T1 \& $\mathrm{T} 2$ heterogeneously hyperintensity with minimal post contrast enhancement s/o late sub-acute bleed causing mass effect on cord with area of altered signal intensity at the level of lesion $\mathrm{f} / \mathrm{s} / \mathrm{o}$ of late sub-acute extra Dural hematoma with myelopathic changes.

On the basis of MRI and clinical data patient was planned for surgery and laminectomy was done with flavum removal. A well-defined firm organized hematoma was seen over the dura which was removed and sent for histological examination which confirmed the presence of well organised hematoma.

In post-operative status patient showed rapid recovery of neurological deficit and was discharged satisfactorily with almost complete recovery of lower limb weakness and bladder bowel incontinence.

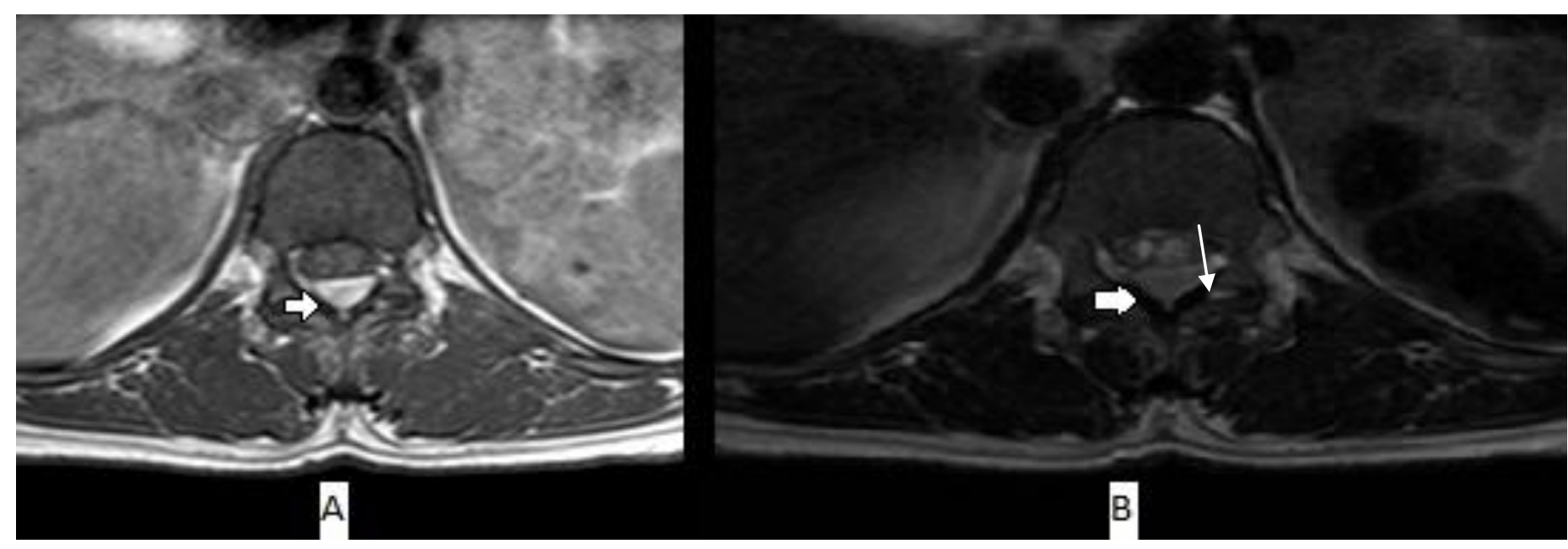

Figure 1: Axial T1 weighted image (A) showing a well-defined heterogeneous hyperintensity (bold arrow) along posterior sub dural space causing mass effect on adjacent cord. On T2 weighted image (B) lesion is heterogeneously hypointense (bold arrow) with altered signal intensity in adjacent cord (Thin arrow).

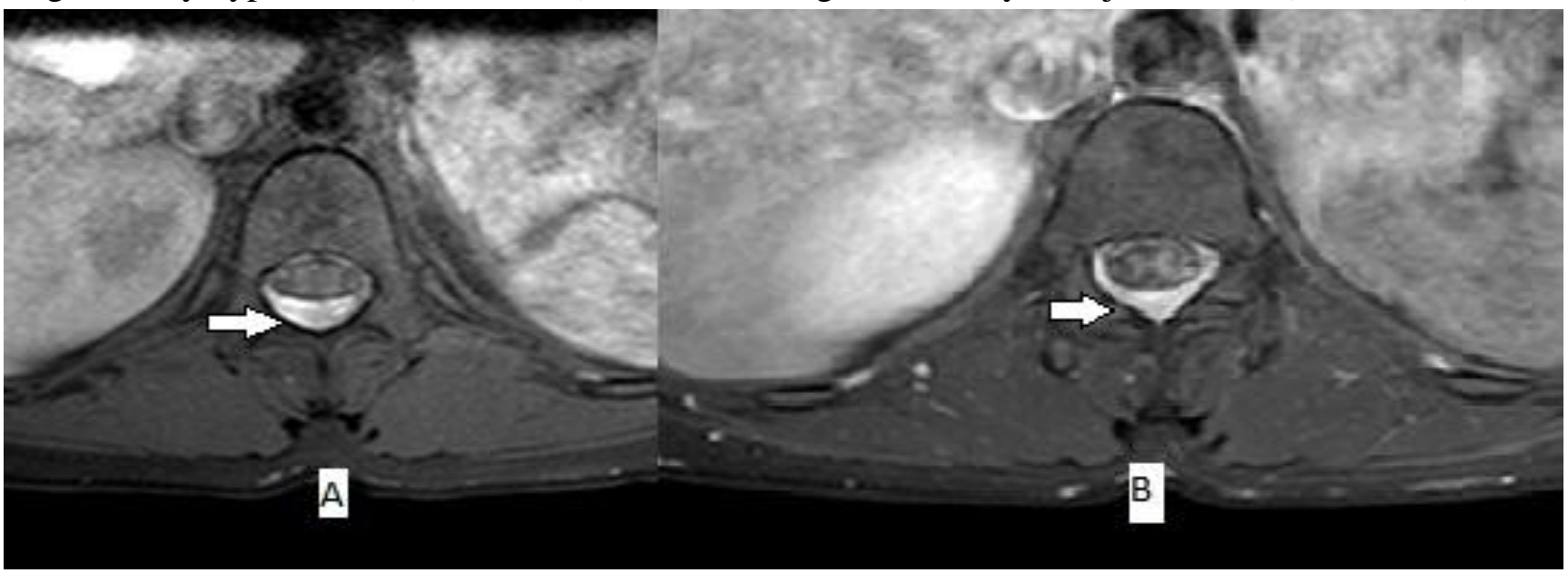

Figure 2: Axial T1 fat suppressed images (A) showing a well-defined heterogeneous hyperintensity along posterior sub dural space causing mass effect on adjacent cord with on post contrast scan (B) showing no significant enhancement. 


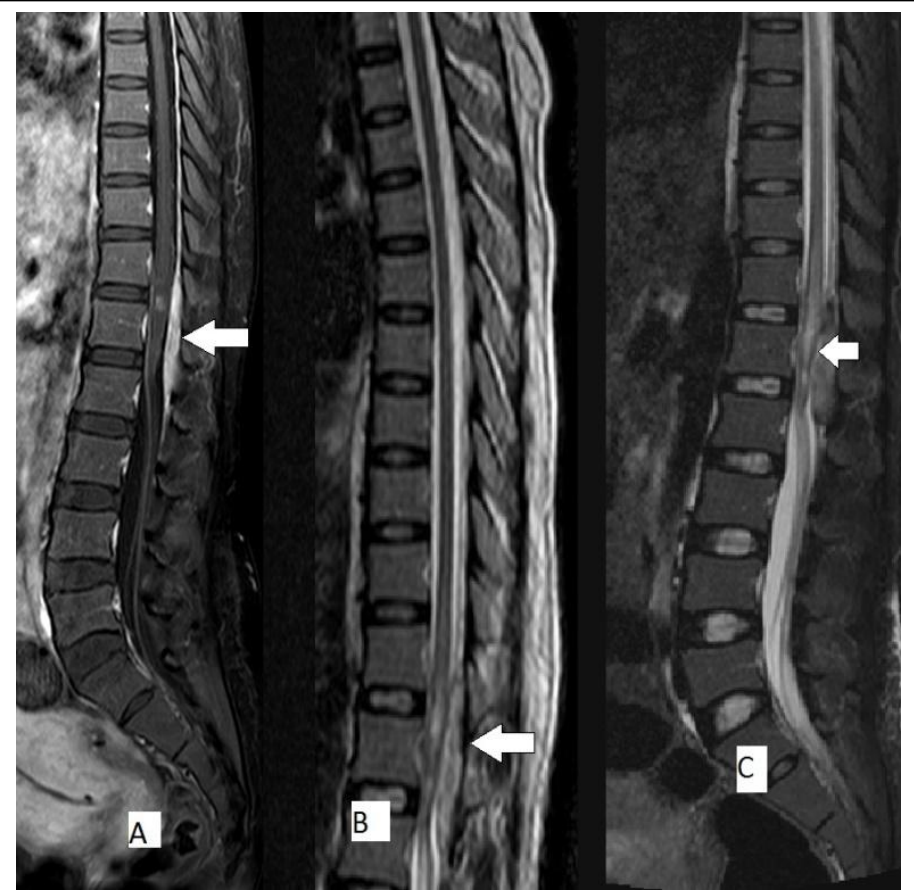

Figure 3: Sagittal T1 (A) and T2 (B) weighted images showing a well-defined altered signal lesion hyperintense (bold arrow) on $\mathrm{T} 1$ and hypointense on T2/STIR (C) images along posterior sub dural space with altered signal in adjacent cord.

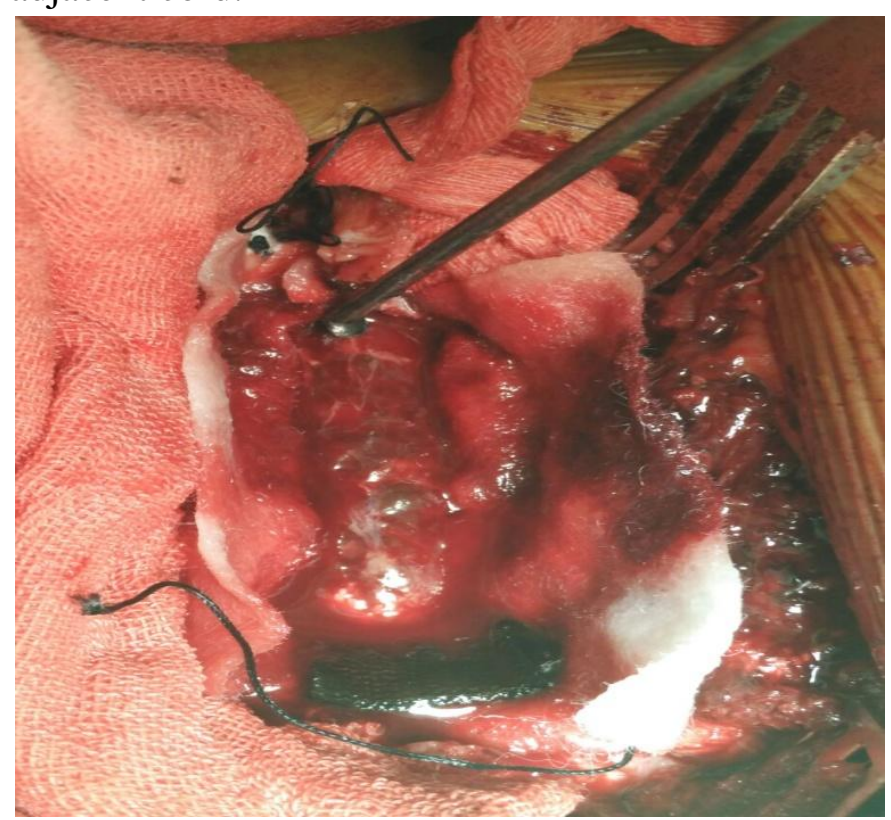

Figure 4: intra-operative picture showing lesion.

\section{DISCUSSION}

Sudden onset paraplegia in postpartum period with back ache \& bladder and bowel involvement without presence of any presiding predisposing factor like trauma, coagulopathy, anti-coagulant therapy, vascular malformation, eclampsia or iatrogenic cause like spinal anaesthesia for labour is extremely rare. Prevalence of SSEH is very low with total approximately 600 cases have been reported in international literature ${ }^{[3]}$ with only 6 cases are been reported in relation to pregnancy.

The origin of blood in SSEH is controversial with a group give thought that it is venous in origin as venous plexus in spine is valve less and any increase in intra-abdominal or intra-thoracic pressure will cause congestion resulting leak of blood through it and resultant hematoma formation, and during pregnancy due to hormonal changes there is further increase in fragility of vessel wall ${ }^{[8]}$. Another group propose a theory of its arterial in origin as arterial bleed can cause spinal compression whereas intrathecal pressure is more than the pressure in venous plexus. ${ }^{[2,4,5,]}$

SSEH is more common in adult males with male to female ratio of $1.4: 1^{[10]}$ and most frequently involve the cervical and cervicodorsal region. Epidural hematoma formation occur dorsal to spinal cord because of tight fixation of dura to vertebral bodies ${ }^{[3]}$. Common causes includes ${ }^{[2]}$ minor trauma, coagulopathy, anticoagulant therapy, vascular malformation, transient vascular hypertension, disc herniation and idiopathic causes which constitute the major cases about 40 to $61 \%$ cases. $^{[10]}$

SSEH typically present with sudden onset backache in neck and interscapular region with progressive neurological deficit hemiplegia or quadriplegia depending on the level of lesion. Currently MRI is been the modality of choice to diagnose SSHE, in subacute stage lesion will be typically well defined biconvex lesion with smooth tapering edges in epidural space showing isointense to hyperintensity on T1WI and hypointense on T2WI with blooming on T2FFE. Depending on the size of hematoma there may be adjacent cord compression ${ }^{[2]}$.

Differential diagnosis of SSHE includes epidural abscess or collection, spinal ischemia, acute disc herniation, spondylitis, transverse myelitis, dissecting aortic aneurysm or even myocardial infarction $^{[6]}$.

Early diagnosis of SSHE with the help of MRI is very use full to localize the lesion and depending 
on the neurological status decision for early surgical intervention ${ }^{[2]}$. The procedure includes decompressive laminectomy and removal of hematoma, dura is opened to exclude sub dural hematoma. Depending on the neurological deficit surgery is planned within 36 hours in case of complete neurological deficit and within 48 hours in case of incomplete neurological deficit ${ }^{[9]}$. Conservative management is also documented in cases where neurological deficit is improving is improving in early phase or in case of coagulopathy. Patient with multilevel of SSHE are difficult to treat operatively. In a study there is diagnostic role of MRI has been defined however there is no definite prognostic role of MRI in predicting the outcome. It is the neurological status of the patient which dictate the line of management, as non-operative treatment ${ }^{[7]}$.

\section{CONCLUSION}

Sudden onset paraplegia in post-partum period in previously healthy female is rarely caused by epidural hematoma. Diagnosis of spontaneous spinal epidural hematoma with the help of MRI helps in early planning for the treatment as early diagnosis and treatment can help in complete reversal of neurological deficits and prevent permanent morbidity.

\section{REFERENCE}

1. Holtås S, Heiling $M$, Lönntoft $M$. Spontaneous spinal epidural hematoma: findings at MR imaging and clinical correlation.Radiology. $1996 \quad$ May; 199(2):409-13.

2. Byung Suck Baek, M.D., Jin Woo Hur, M.D., Ki Young Kwon, M.D., and Hyun Koo Lee, M.D. Spontaneous Spinal Epidural Hematoma. J Korean Neurosurg Soc. 2008 Jul; 44(1): 40-42.

3. Singh H, Aggarwal Shalini, Gupta Rajat, Yadav RK. Spontaneous Spinal Epidural Haemorrhage. An Unusual Cause of Paraplegia. JIACM 2008; 9(2): 136-9.
4. Beatty RM, Winsten KR. Spontaneous cervical epidural hematoma. A consideration of aetiology. J Neurosurg 1984;61: 143-8.

5. Farias JP, Lima JA, Antunes JL. Subacute cervical epidural hematomas. SurgNeurol1994; 42: 414-6.

6. Leandro U Taniguchi1, Felix H Pahl, José ED Lúcio, Roger S Brock, Marcos QT Gomes, TarsoAdoni, Victor CC Fiorini, Rodrigo C Carvalho, Eli F Evaristo, Eduardo G Mutarelli and Guilherme Schettino; Complete motor recovery after acute paraparesis caused by spontaneous spinal epidural hematoma: case report; BMC Emergency Medicine 2011, 11:10

7. Melanie B. Fukui, Amar S. Swarnkar, and Robert L. Williams; Acute Spontaneous Spinal Epidural Hematomas; AJNR Neuroradiol20:1365-1372, August 1999

8. Hisako Fujimaki, Toshiyuki Nakazawa, Masaki Ueno, Takayuki Imura, Wataru Saito, NaonobuTakahira and Masashi Takaso; Spinal subarachnoid hematoma in a woman with HELLP syndrome: a case report; Journal of Medical Case Reports 2012, 6:152

9. Coulibaly O, Habibou M, Gana R, Maaqili R, Bellakhdar F.; Spontaneous spinal epidural haematoma causing paraplegia: a case report

10. Chen CL, Lu CH, Chen NFl: Spontaneous spinal epidural haematoma presenting with quadriplegia after sit-ups exercise. American Journal of Emergency Medicine 2009; 27: 1170.e3-1170.e7. 